\title{
STAT3 Decoy Oligonucleotide
}

National Cancer Institute

\section{Source}

National Cancer Institute. STAT3 Decoy Oligonucleotide. NCI Thesaurus. Code C78822.

A double-stranded 15-mer oligonucleotide, corresponding closely to the signal transducer and activator of transcription 3 (STAT3) response element within the c-fos promoter, with potential antineoplastic activity. ST AT 3 decoy oligonucleotide binds specifically to activated STAT 3 and blocks binding of STAT 3 to DNA sequences on a variety of STAT3-responsive promoters, which results in the inhibition of STAT 3mediated transcription and, potentially, the inhibition of tumor cell proliferation. STAT 3 is constitutively activated in a variety of cancers including squamous cell carcinoma of the head and neck, contributing to the loss of cell growth control and neoplastic transformation. 